\title{
CONCATENATED SPACE TIME BLOCK CODES AND TCM, TURBO TCM, CONVOLUTIONAL AS WELL AS TURBO CODES
}

\author{
T. H. Liew, J. Pliquett, B. L. Yeap, L-L. Yang and L. Hanzo \\ Dept. of ECS., Univ. of Southampton, SO17 1BJ, UK. \\ Tel: +44-703-593 125, Fax: +44-703-593045 \\ Email: th197r@ecs.soton.ac.uk,1h@ecs.soton.ac.uk \\ http://www-mobile.ecs.soton.ac.uk
}

\begin{abstract}
Space-time block codes provide substantial diversity advantages for multiple transmit antenna systems at a low decoding complexity. In this paper, we concatenate space-time codes with Convolutional Codes (CC), Turbo Convolutional codes (TC), Turbo BCH codes (TBCH), Trellis Coded Modulation (TCM) and Turbo Trellis Coded Modulation (TTCM) schemes for achieving a high coding gain. The associated performance and complexity of the the coding schemes is compared.
\end{abstract}

\section{INTRODUCTION}

The third generation (3G) mobile communication standards are expected to support a wide range of bearer services, spanning from voice to high-rate data services supporting rates of at least $144 \mathrm{~kb} / \mathrm{s}$ in vehicular, $384 \mathrm{~kb} / \mathrm{s}$ in outdoorto-indoor and $2 \mathrm{Mb} / \mathrm{s}$ in indoor as well as picocellular applications [1]. In an effort to support such high rates, the capacity of band-limited wireless channels can be increased by employing multiple antennas. Recently, different transmit diversity techniques have been introduced, in order to provide diversity gain for MSs by upgrading the BSs. In [2], Tarokh et al. proposed space-time trellis coding by jointly designing the channel coding, modulation, transmit diversity and the optional receiver diversity. The proposed space-time trellis codes perform extremely well at the cost of high complexity. In addressing the issue of decoding complexity, Alamouti [3] discovered a remarkable scheme for transmissions using two transmit antennas. A simple decoding algorithm was introduced, which can be generalised to an arbitrary number of receive antennas. This scheme is significantly less complex, than space-time trellis coding using two transmit antennas, although there is a loss in performance [4]. Despite the associated performance penalty, Alamouti's scheme is appealing in terms of simplicity and performance. This proposal motivated Tarokh

This work has been performed in the framework of the PanEuroepan IST project IST-1999-12070 (TRUST), which is partly funded by the European Union. The authors would like to acknowledge the contributions of their colleagues, although the views expressed are those of the authors.

The financial support of the EPSRC, Swindon, UK is also gratefully acknowledged.

Globecom'2000, San Francisco, USA et al. $[4,5]$ to generalise the scheme to an arbitrary number of transmit antennas, leading to the concept of spacetime block codes. Space-time block codes were designed for achieving the maximum diversity order of $n \times m$ for $n$ transmit and $m$ receive antennas. However, they were not designed for achieving additional coding gain. Hence, in this contribution, we combine space-time block codes with Convolutional Codes (CC) $[6,7]$, Turbo Convolutional (TC) codes $[7,8]$, Turbo BCH codes (TBCH) [9], Trellis Coded Modulation (TCM) [10] and Turbo Trellis Coded Modulation (TTCM) [11], in order to achieve additional coding gains. The performance and complexity of the different schemes will be studied comparatively.

\section{SYSTEM OVERVIEW}

The schematic of the proposed concatenated space-time block codes and the different turbo coding schemes is shown in Figure 1. The information bits will be encoded by different coding schemes, as shown in Figure 1. In Table 1,

\begin{tabular}{|l|c|c|c|c|}
\hline Code & $\begin{array}{c}\text { Rate } \\
R\end{array}$ & $\begin{array}{c}\text { Octal } \\
\text { generator } \\
\text { polynomial }\end{array}$ & $\begin{array}{c}\text { No. } \\
\text { of } \\
\text { states }\end{array}$ & $\begin{array}{c}\text { Modu- } \\
\text { lation } \\
\text { Scheme }\end{array}$ \\
\hline $\mathrm{CC}(2,1,9)$ & $1 / 2$ & 561,753 & 256 & $64 \mathrm{QAM}$ \\
\hline $\mathrm{TC}(2,1,3)$ & $1 / 2$ & 7,5 & 4 & $64 \mathrm{QAM}$ \\
\hline $\mathrm{TC}(2,1,4)$ & $1 / 2$ & 13,15 & 8 & $64 \mathrm{QAM}$ \\
\hline $\mathrm{TC}(2,1,5)$ & $1 / 2$ & 23,35 & 16 & $64 \mathrm{QAM}$ \\
\hline $\mathrm{TBCH}(31,26)$ & $26 / 36$ & 45 & 32 & $16 \mathrm{QAM}$ \\
\hline TCM & $3 / 4$ & $101,16,64$ & 64 & 16QAM \\
\hline TTCM & $3 / 4$ & $23,2,4,10$ & 16 & $16 \mathrm{QAM}$ \\
\hline
\end{tabular}

Table 1: Table of different channel coders in Figure 1.

we show the coding parameters of the investigated coding schemes and the modulation schemes employed. The modulation scheme is chosen such that the throughput of the all schemes is fixed at 3 Bits Per Symbol (BPS). The first coding scheme, namely the convolutional code is denoted by $\operatorname{CC}(n, k, K)$, where $n, k$ and $K$ denote the number of coded bits, input bits and the constraint length, respectively. Two identical recursive systematic convolutional codes (RSC) are employed in the turbo convolutional code, which is denoted by $\mathrm{TC}(n, k, K)$. The half-rate TC codes are generated by puncturing the parity bits alternatively [8]. Besides convolutional codes, $\mathrm{BCH}$ codes are used as the component codes in the turbo codes. The information and 


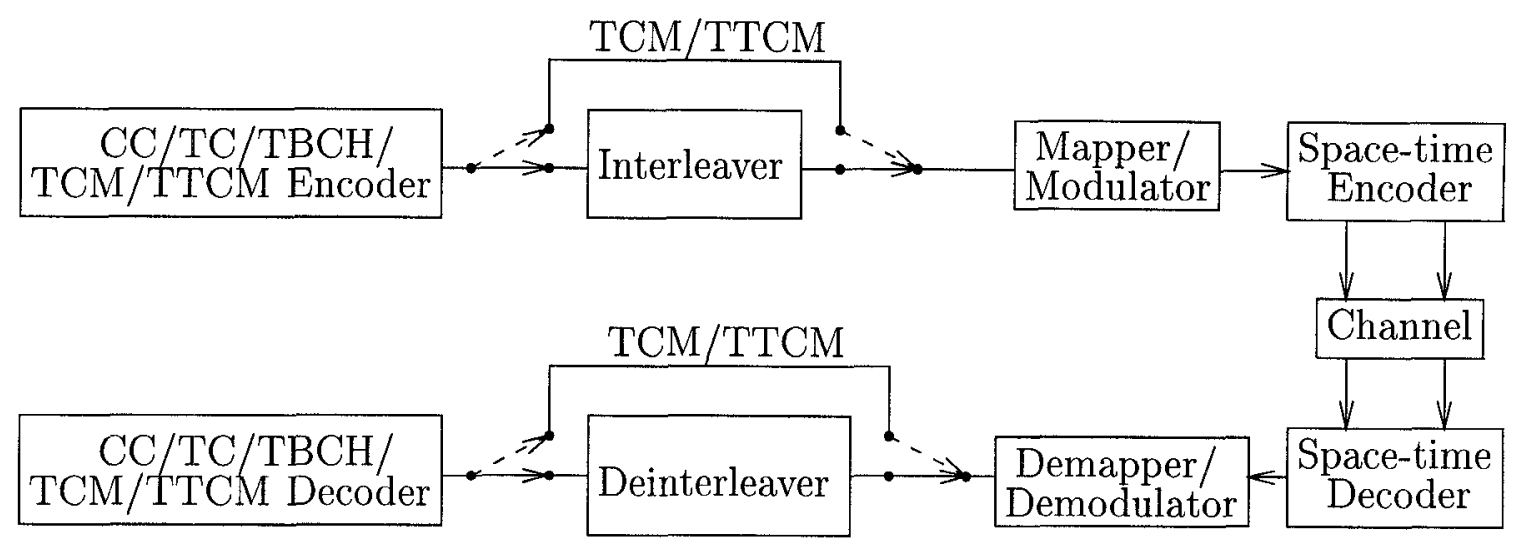

Figure 1: System overview of space-time block codes and different coding schemes.

parity bits of the $\mathrm{BCH}(31,26)$ component encoders are not punctured, which results in a code rate of $R=0.72$ [9]. The $\operatorname{TBCH}(n, k)$ codes have been shown for example by Hagenauer [9] to perform impressively at near-unity coding rates, although at high complexity. Finally, we also investigate Trellis Coded Modulation (TCM) [10] and Turbo Trellis Coded Modulation (TTCM) [11]. Note that $\mathrm{CC}(2,1,9)$ and $\mathrm{TC}(2,1,4)$ are both proposed in the $3 \mathrm{G}$ mobile communication standards $[1,7]$.

As seen in Figure 1, only the output bits of the CC, TC and TBCH schemes are passed to the interleaver, while TCM and TTCM use no interleaving. We apply random separation based interleaving [12] for dispersing the effects of bursty errors. The output bits of the TCM and TTCM scheme are passed directly to the mapper/modulator in Figure 1, where we employed two different mapping techniques. Gray mapping assisted 16-level Quadrature Amplitude Modulation (16QAM) and 64-level Quadrature Amplitude Modulation (64QAM) is used for the CC, TC and TBCH schemes, whereas set partitioning $[10,11]$ assisted 16QAM is utilised for the TCM and TTCM schemes.

Following the mapper/modulator, the coded symbols are passed to the space-time block encoder, as shown in Figure 1. A space-time block code is defined by a $p \times n$ transmission matrix $\mathbf{G}$, where the entries of the matrix are linear combinations of the input symbols $x_{1}, x_{2}, \ldots, x_{k}$ and their conjugates. The number of transmitter antennas is $n$ and the code span is $p$. The $p \times n$ matrix $\mathbf{G}$ - which defines the space-time block code - is based on a complex generalised orthogonal design, as defined in [3,4]. In our system, we used the simplest space-time block code defined in $[3,4]$ as:

$$
\mathbf{G}_{2}=\left(\begin{array}{cc}
x_{1} & x_{2} \\
-x_{2}^{*} & x_{1}^{*}
\end{array}\right)
$$

The code rate of the space-time code is given by $k / p$, and hence in this example the code rate is unity. All symbols in the same row of the matrix $\mathbf{G}$ are transmitted simultaneously from $n$ different transmit antennas, while all entries in the same column are transmitted from the same antenna in $p$ successive transmission instants. Apart from the space-time block code $\mathbf{G}_{2}$ of Figure 1, we also consider the performance of a range of other lower-rate space-time block codes, namely that of $\mathbf{G}_{3}, \mathbf{G}_{\mathbf{4}}, \mathbf{H}_{3}$ and $\mathbf{H}_{4}$ proposed in $[4,5]$. The space-time codes $R=1 / 2 \mathbf{G}_{3}$ and $R=3 / 4 \mathbf{H}_{3}$ have three transmit antennas, while the codes $R=1 / 2 \mathbf{G}_{4}$ and $R=3 / 4 \mathrm{H}_{4}$ have four transmit antennas, respectively.
The number of receive antennas constitutes a design parameter, which was fixed to one in our system. At high bit rates, the channel does not change significantly for $p$ consecutive symbols. Therefore, the orthogonality of the spacetime code matrix enables us to separate the signals $x_{1}$ and $x_{2}$ transmitted from the different antennas. This then allows us to invoke the Log-MAP algorithm [13] for the independent decoding of the signals received from the different antennas. The soft outputs associated with the received bits or symbols are passed to the deinterleaver or TCM/TTCM decoder, respectively, as seen in Figure 1. The deinterleaved soft outputs of the received bits will then be passed to the $\mathrm{CC} / \mathrm{TC} / \mathrm{TBCH}$ decoders. All of the turbo schemes studied apply the Log-MAP decoding algorithm and the number of iterations are fixed at eight. The CC and TCM decoder employs the soft decision Viterbi algorithm $[6,10]$.

\section{SIMULATION RESULTS}

All simulation results were obtained over uncorrelated or synonymously - perfectly interleaved narrow-band Rayleigh fading channels. This assumption does not contradict to requiring a constant channel magnitude and phase over $p$ consecutive symbols, since upon applying a high interleaving depth the channel's fading envelope can indeed be near uncorrelated. We assumed that the narrow-band fading amplitudes received from each transmitter antenna were mutually uncorrelated Rayleigh distributed processes. It was also assumed that the fading amplitude was constant across $p$ (number of rows in the space-time matrix) consecutive symbols. The average signal power received from each transmitter antenna was the same. Furthermore, we assumed that the receiver had a perfect estimate of the channel's fading amplitudes.

In [5], Tarokh et al. have shown that without channel coding the performance of the unity-rate space-time code $\mathbf{G}_{2}$ is inferior to the lower rate space-time codes, namely to that of $\mathbf{G}_{3}, \mathbf{G}_{\mathbf{4}}, \mathbf{H}_{3}$ and $\mathbf{H}_{4}$. Since the space-time code $\mathbf{G}_{2}$ has a unity code rate, half-rate turbo codes can be employed to improve the performance of the system, while maintaining the same throughput as the other space-time codes. In Figure 2, we compare the performance of the half-rate $\mathrm{TC}(2,1,4)$ code concatenated with the space-time code $\mathbf{G}_{2}$ and with the space-time block codes $\mathbf{G}_{\mathbf{3}}, \mathbf{G}_{4}, \mathbf{H}_{3}$ and $\mathbf{H}_{4}$. Both the space-time codes $\mathbf{G}_{4}$ and $\mathbf{H}_{4}$ have a diversity gain of four and a code rate of $\frac{1}{2}$ and $\frac{3}{4}$, respectively. From Figure 2, we can see that a huge performance improvement is achieved by concatenating the space-time 


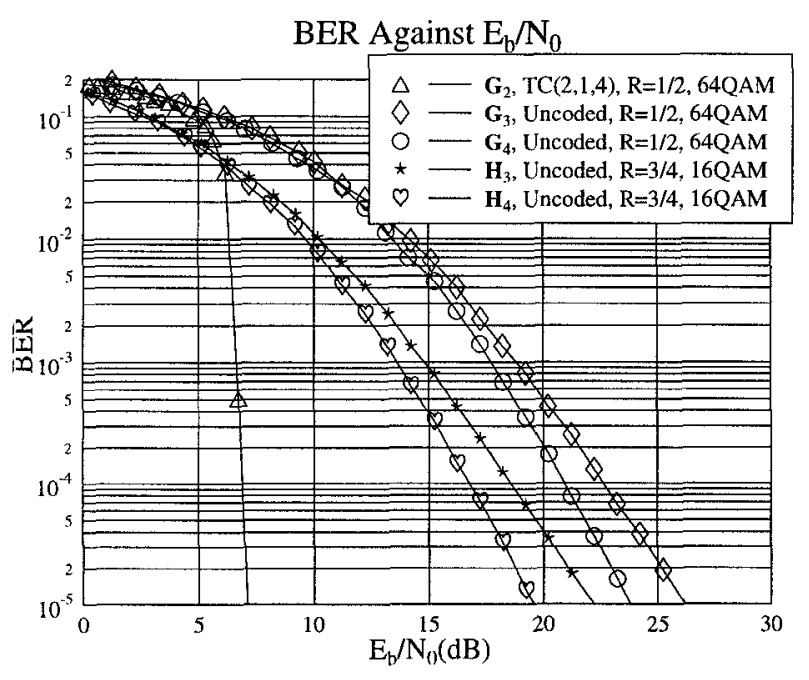

Figure 2: Performance comparison of the half-rate TC $(2,1,4)$ code concatenated with the space-time code $\mathbf{G}_{2}$ and the space-time block codes $\mathbf{G}_{3}, \mathbf{G}_{4}, \mathbf{H}_{3}$ and $\mathbf{H}_{4}$. The coding parameters are shown in Table 1 . All simulation results were obtained at an effective throughput of 3 BPS over uncorrelated Rayleigh fading channels.

code $\mathbf{G}_{2}$ with the half-rate code $\mathrm{TC}(2,1,4)$. At a BER of $10^{-5}$ this concatenated scheme attains a coding gain of 16 $\mathrm{dB}$ and $13 \mathrm{~dB}$ as compared to the space-time codes $\mathbf{G}_{4}$ and $\mathbf{H}_{4}$, respectively. This clearly shows that it is better to invest the parity bits associated with the code-rate reduction in the concatenated turbo code, rather than in non-unity-rate space-time codes. From Figure 2 we can coriclude that the reduction in coding rate is best assigned to turbo codes, rather to space-time codes. Therefore, in all our forthcoming simulations, all channel codecs are concatenated with the unity-rate space-time code $\mathbf{G}_{2}$, instead of the non-unity-rate space-time codes $\mathbf{G}_{3}, \mathbf{G}_{4}, \mathbf{H}_{3}$ and $\mathbf{H}_{4}$.

In Figure 3 we portrayed the performance of the $\mathrm{CC}(2,1,9)$, TC $(2,1,4)$, TBCH $(31,26)$, TCM and TTCM schemes on the basis of a constant throughput of 3 BPS, regardless of their cocling rates. The associated coding parameters are shown in Table 1. The simulation results were obtained by employing the space-time code $\mathbf{G}_{2}$ over uncorrelated Rayleigh fading channels. From Figure 3 we observed that the turbo schemes TC, TBCH and TTCM outperform the conventional CC and TCM schemes. In [12], we have shown that at a throughput of 2 BPS, TC schemes using 16QAM outperform TBCH and TTCM schemes using 8-level Phase Shift Keying (8PSK). However, in Figure 3 the performances of the TC, TBCH and TTCM schemes are similar. This is because, in order to maintain a throughput of 3 BPS, 64QAM has to be employed in the systems using the halfrate TC $(2,1,4)$ code. The constellation points in 64QAM are more densely packed than those of 16QAM. Therefore, the rather vulnerable 64QAM appears to over-stretch the coding power of the half-rate $\mathrm{TC}(2,1,4)$ code. Hence at a BER of $10^{-5}$ there is no obvious performance gain over the TB.CH $(31,26)$ and TTCM schemes, which were applied in conjunction with 16QAM. However, in the next section we will show that the $\mathrm{TC}(2,1,4)$ code is a better choice compared to the other turbo schemes, when considering the decoding complexity.

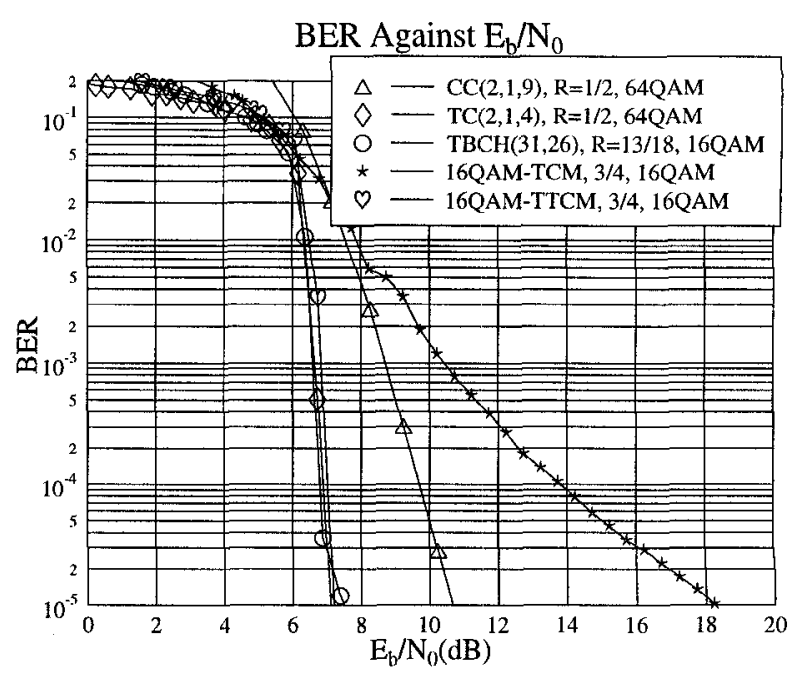

Figure 3: Performance comparison between different $\mathrm{CC}(2,1,9), \mathrm{TC}(2,1,4), \mathrm{TBCH}(31,26)$, TCM and TTCM schemes where the coding parameters are shown in Table 1. All simulation results were obtained by employing the space-time code $\mathbf{G}_{2}$ at an effective throughput of $\mathbf{3}$ BPS over uncorrelated Rayleigh fading channels.

\section{PERFORMANCE VERSUS COMPLEXITY}

In this section we are going to address the complexity issues of the proposed system. We will mainly focus on the relative complexity of the proposed channel decoders, rather than attempting to determine their exact complexity. Therefore, in order to simplify our comparative study, several assumptions are made. In our simplified approach the complexity of the whole system is deemed to depend only on that of the channel decoders. In other words, the complexity associated with the modulator, demodulator, space-time encoder and decoder as well as channel encoders are assumed to be insignificant as compared to the complexity of channel decoders. We adopted the approach of [11], where the number of trellis transitions per information data bit was used as the basis of our comparison.

For the binary convolutional code $\mathrm{CC}(2,1, K)$, two trellis transitions diverge from each of the $2^{K-1}$ states. Hence, we can approximate the complexity of a $\mathrm{CC}(2,1, K)$ code as:

$$
\operatorname{comp}\{\mathrm{CC}(2,1, \mathrm{~K})\}=2^{K} .
$$

The number of trellis transitions for the Log-MAP decoding algorithm is assumed to be three times higher, than that of the conventional Viterbi algorithm, since the Log-MAP algorithm has to perform forward as well as backward recursion and soft output calculations, which results in traversing through the trellis three times. For TC codes we apply the Log-MAP decoding algorithm for iterative decoding assisted by the two component decoders. Upon taking into account the number of turbo decoding iterations as well, the complexity of TC decoding is then approximated by:

$$
\operatorname{comp}\{T C(2,1, K)\}=3 \times 2^{K+1} \times \text { No. of Iterations (.3) }
$$

In TCM we construct a non-binary decoding trellis [10]. The TCM schemes of Table 1 have $2^{B P S-1}$ trellis branches diverging from each trellis state, where $B P S$ is the number 
of transmitted bits per modulation symbol. However, for each trellis transition we would have $B P S-1$ transmitted information data bits, since the TCM encoder typically adds one parity bit per non-binary symbol. Therefore, we can estimate the complexity of the proposed TCM schemes as:

$$
\operatorname{comp}\{\mathrm{TCM}\}=2^{B P S-1} \times \frac{\text { No. of States }}{B P S-1},
$$

where the number of states is shown in Table 1. Similarly to TC, TTCM consists of two TCM codes and the LogMAF decoding algorithm [11] is employed for their iterative decoding. The associated TTCM complexity is then estimated as:

$$
\operatorname{comp}\{\mathrm{TTCM}\}=\frac{3 \times 2^{B P S} \times \text { No. of States }}{B P S-1} \times
$$

For $\operatorname{TBCH}(n, k)$ codes the complexity calculation is not as straight forward as in the previous cases. Its component codes are $\operatorname{BCH}(n, k)$ codes and the decoding trellis can be divided into three sections. Assuming that $k>n-k$, for every decoding instant $j$ the number of trellis states is given as:

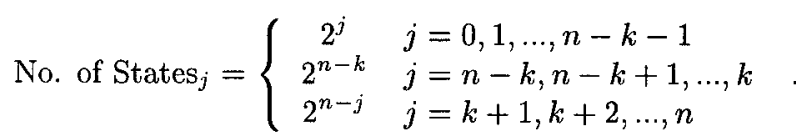

Upon using the relationship $\sum_{j=0}^{n-k-1} 2^{j}=\sum_{j=k+1}^{n} 2^{n-j}=$ $2^{n-k}-1$ and the approximation that $2^{n-k}-1 \approx 2^{n-k}$, we can write the number of decoding trellis states per information data bit as:

$$
\begin{aligned}
\text { No. of States } & =\frac{2 \times 2^{n-k}+\{k-(n-k)\} \times 2^{n-k}}{k} \\
& =\frac{(2 k-n+2) \times 2^{n-k}}{k} .
\end{aligned}
$$

Having derived the number of decoding trellis states per information data bit, we can approximate the complexity of the TBCH codes as:

$$
\operatorname{comp}\{\operatorname{TBCH}(n, k)\}=\frac{3 \times(2 k-n+2) \times 2^{n-k+2}}{k} \times
$$

since two $\mathrm{BCH}$ decoders are employed and there are two transitions leaving each state. Having derived the approximation of the decoding complexity, we now compare the performances of the various coding schemes in the light of their decoding complexity.

Figure 4 shows the corresponding coding gain versus complexity curves for the $\mathrm{CC}(2,1, K), \mathrm{TC}(2,1,4), \mathrm{TBCH}(31,26)$ and TTCM schemes, where the coding parameters are shown in Table 1 . The estimated complexity was obtained by using Equations 2 to 8 . Again, all simulation results were obtained upon employing the space-time code $\mathbf{G}_{2}$ using one receiver over uncorrelated Rayleigh fading channels at an effective throughput of 3 BPS. The increased complexity of the turbo schemes is incurred by increasing the number of iterations from 1 to 10 . For the convolutional codes the constraint length $K$ is varied from 3 to 10 , which results

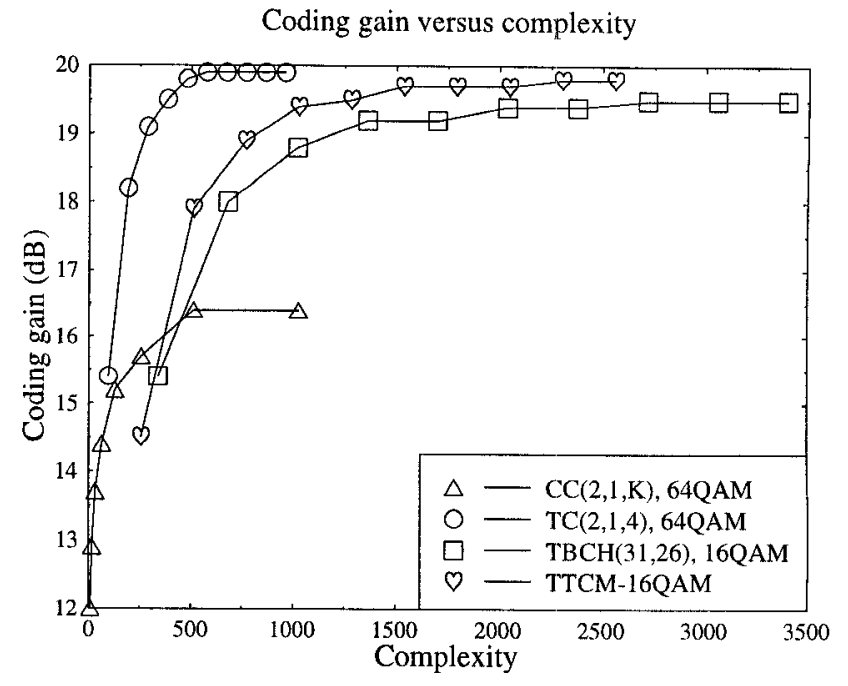

Figure 4: Coding gain versus complexity for the $\mathrm{CC}(2,1, K)$, $\mathrm{TC}(2,1,4)$, TBCH $(31,26)$ and TTCM schemes where the coding parameters are shown in Table 1. All simulation results were obtained upon employing space-time code $\mathbf{G}_{2}$ using one receiver over uncorrelated Rayleigh fading channels at an effective throughput of 3 BPS

in their increased complexity. The generator polynomials of the $\mathrm{CC}(2,1, K)$ code, where $K=3 \ldots 10$, are given in [6] and they define the corresponding maximum free distance codes. In Figure 4 we can see that there is a steep increase in the coding gain achieved by the $\mathrm{TC}(2,1,4)$ code, as the complexity is increased. However, the TC(2,1,4) scheme asymptotically achieves a maximum coding gain of approximately $20 \mathrm{~dB}$. In order to achieve a coding gain of $18 \mathrm{~dB}$, the TTCM and TBCH $(31,26)$ arrangements would require an approximately 3 and 4 times higher computational power compared to the $\mathrm{TC}(2,1,4)$ code. From Figure 4 we can clearly see that TC codes are the most attractive ones of all the channel codes studied in conjunction with the spacetime code $\mathbf{G}_{2}$ code, offering an impressive coding gain at a moderate decoding complexity.

Having shown that TC $(2,1,4)$ is the best coding scheme, we now compare the performance and complexity of TC codes having different constraint length $K$. Figure 5 shows the (a) coding gain versus the number of iterations and (b) the coding gain versus complexity for the $\mathrm{TC}(2,1,3)$, $\mathrm{TC}(2,1,4)$ and $\mathrm{TC}(2,1,5)$ codes, where the coding parameters used are shown in Table 1 . The coding gain defined as the $E_{b} / N_{0}$ difference, expressed in decibels, at a $\mathrm{BER}=10^{-5}$ between the various channel coded and the uncoded system having the same throughput, while using the space-time code $\mathbf{G}_{2}$. All simulation results were obtained upon employing the space-time code $\mathbf{G}_{2}$ using one receiver and 64QAM over uncorrelated Rayleigh fading channels at an effective throughput of 3 BPS. We can see from Figure 5(a) that there is a huge performance improvement of approximately $3-4 \mathrm{~dB}$ between the first and second turbo decoding iteration. However, the further coding gain improvements become smaller, as the number of iterations increases. It can be seen from the figure that the performance of turbo codes does not significantly improve after 8 iterations, as indicated by the rather flat coding gain curve. Figure 5(a) also shows that as we increase the constraint 
Coding gain versus number of iterations

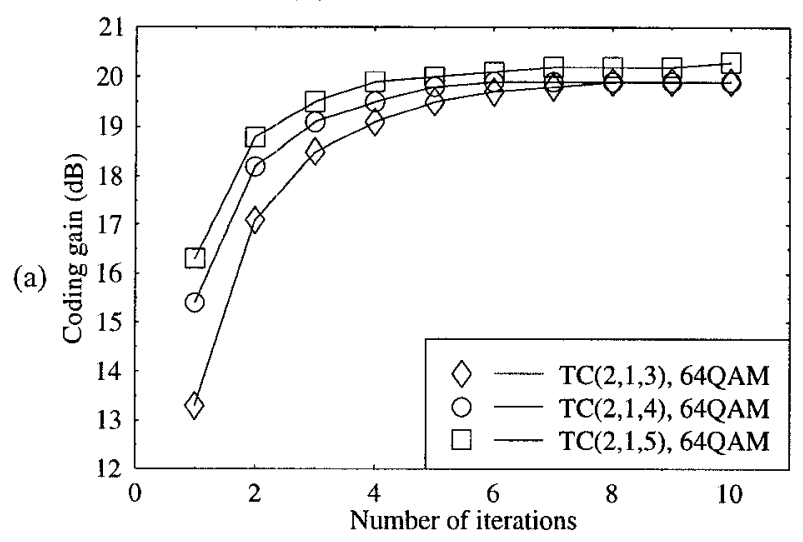

Coding gain versus complexity

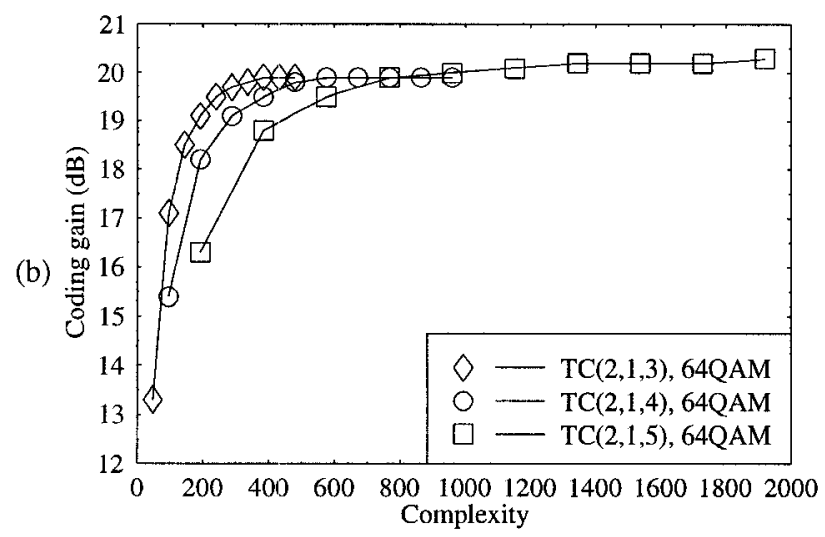

Figure 5: Coding gain versus (a) the number iterations and versus (b) complexity for the $\mathrm{TC}(2,1,3), \mathrm{TC}(2,1,4)$ and $\mathrm{TC}(2,1,5)$ codes, where the coding parameters are shown in Table 1. All simulation results were obtained upon employing the space-time code $\mathbf{G}_{2}$ using one receiver and $64 \mathrm{QAM}$ over uncorrelated Rayleigh fading channels at an effective throughput of 3 BPS.

length $K$ of the turbo codes from 3 to 5 , the associated performance improves.

In Figure 5 (b) the coding gains of the various turbo codes using different number of iterations were compared on the basis of their complexity. We can see from Equation 3 that the complexity of turbo codes depends exponentially on the constraint length $K$, but only linearly on the number of iterations. From Figure 5(b), we can see that the estimated complexity of the constraint length five $\mathrm{TC}(2,1,5)$ code ranges from approximately 200 to 2000 , when using one to ten iterations. By contrast, the complexity of the constraint length three $\mathrm{TC}(2,1,3)$ scheme ranges only from approximately 50 to 500 upon invoking one to ten iterations. This clearly shows that the complexity of the turbo codes is dominated by the constraint length $K$. Figure $5(\mathrm{~b})$ also shows that the coding gain curve of the $\mathrm{TC}(2,1,3)$ code saturates faster, which is demonstrated by the steep increase in coding gain as the complexity increases. For achieving the same coding gain of $19 \mathrm{~dB}$, we can see that the $\mathrm{TC}(2,1,3)$ scheme requires the lowest complexity. We would require 23 times higher computational power for the $\mathrm{TC}(2,1,5)$ code to achieve the above coding gain of $19 \mathrm{~dB}$.

\section{CONCLUSION}

In this contribution we investigated the concatenation of different channel coding schemes in conjunction with spacetime block codes. We first compared the performance of the half-rate $\mathrm{TC}(2,1,4)$ code concatenated with the space-time code $\mathbf{G}_{2}$ and with the space-time block codes $\mathbf{G}_{3}, \mathbf{G}_{4}, \mathbf{H}_{3}$ and $\mathbf{H}_{4}$. We concluded that the reduction in coding rate is best assigned to turbo channel codes, rather to space-time codes. Then, the performance of different channel coding schemes were compared on the basis of a constant throughput of 3 BPS. The turbo schemes TC, TBCH and TTCM achieved similar performance, while outperforming the noniterative decoding schemes CC and TCM. However, by considering the associated decoding complexity, the half-rate TC codes gave the best coding gain at a moderate complexity. Our future work will be focused on a similar study of space-time codes in the context of dispersive channels and Code Division Multiple Access (CDMA) transmissions.

\section{REFERENCES}

[1] P. Chaudhury, "The 3GPP Proposal for IMT-2000", IEEE Communications Magazine, Vol. 37, no. 12, pp. 72-81, December 1999.

[2] V. Tarokh, N. Seshadri and A. R. Calderbank, "Space-Time Codes for High Data Rate Wireless Communication: Performance Criterion and Code Construction", IEEE Transactions on Information Theory, Vol. 44, no. 2 pp. 744-765, March 1998.

[3] S. M. Alamouti, "A Simple Transmit Diversity Technique for Wireless Communications", IEEE JSAC, pp. 1451-1458, October 1998.

[4] V. Tarokh, H. Jafarkhani and A. R. Calderbank, "SpaceTime Block Codes from Orthogonal Designs", IEEE Transactions on Information Theory, pp. 1456-1467, July 1999.

[5] V. Tarokh, H. Jafarkhani and A. R. Calderbank, "SpaceTime Block Coding for Wireless Communications: Performance Results", IEEE JSAC, pp. 451-460, March 1999.

[6] J. Proakis, "Digital Communications", McGraw-Hill International Editions 1995.

[7] 3GPP, "Multiplexing and Channel Coding (TDD)", 3G TS 25.222, http://www.3gpp.org

[8] C. Berrou and A. Glavieux, "Near Optimum Error Correcting Coding and Decoding: Turbo-Codes", IEEE Transactions on Communications, Vol. 44, no. 10, pp. 1261-1271, October 1996.

[9] J. Hagenauer, E. Offer and L. Papke, "Iterative Decoding of Binary Block and Convolutional Codes", IEEE Transactions on Information Theory, Vol. 42, no. 2, pp. 429-445, March 1996.

[10] G. Ungerboeck, "Trellis-Coded Modulation with Redundant Signal Sets Part I: Introduction", IEEE Communications Magazine, Vol. 25, no. 2, pp. 5-11, February 1987.

[11] P. Robertson and T. Worz, "Bandwidth-Efficient Turbo Trellis-Coded Modulation Using Punctured Component Codes", IEEE JSAC, pp. 206-218, February 1998.

[12] T. Liew, J. Pliquett, B. Yeap, L-L. Yang and L. Hanzo, "Comparative Study of Space Time Block Codes and Various Concatenated Turbo Coding Schemes", in Proc. of PIMRC 2000, London, UK.

[13] G. Bauch, "Concatenation of Space-Time Block Codes and Turbo-TCM", Proc IEEE ICC, Vancouver, Canada pp .1202-1206, June 1999. 